Series A

I. MATHEMATICA

394

\title{
ZUR APPROXIMATION ALGEBRAISCHER ZAHLEN
}

VON

SEPPO HYYRÖ 
Am 14 Oktober 1966 vorgelegt von P. J. Myrberg und K. Inkeri 


\section{Zur Approximation algebraischer Zahlen}

1. Einleitung. Das berühmte »zehnte» Problem von HILbert ist (siehe z.B. [3]), einen endlichen Algorithmus für die vollständige Lösung der diophantischen Gleichung

$$
f(x, y)=0
$$

$\mathrm{zu}$ finden, wobei $f(x, y)$ ein gegebenes Polynom mit ganzen rationalen Koeffizienten bezeichnet. Es würde einen wichtigen Fortschritt der Untersuchung des erwähnten Problems bedeuten (das Problem würde nämlich gelöst z.B. in dem Fall, dass die höchsten Glieder ein homogenes Polynom vom Grade $n$ mit keinen mehrfachen linearen Teilern bilden, während die Glieder vom Grade $n-1$ und $n-2$ fehlen), wenn man die folgende Aufgabe lösen könnte:

Sind eine irrationale algebraische Zahl $\vartheta$ und reelle Zahlen $C, \lambda$ mit $C>0, \lambda>2$ gegeben, so soll man explizit eine Schranke $G=G(\vartheta, \lambda, C)$ angeben derart, dass die Ungleichung

$$
|\vartheta-u| v \mid<C / v^{\lambda}
$$

keine Lösungen in ganzen rationalen $u, v$ mit $(u, v)=1, v>G$ hat.

Wie in der Literatur manchmal konstatiert ist, scheint die THUESiEgEL-Rothsche Methode keine Lösung für diese Aufgabe im allgemeinen Fall zu geben. Wir ([7], [8]) haben jedoch neulich gezeigt (vgl. auch BAKER [1], [2]), dass die Aufgabe gelöst werden kann, wenn $\vartheta$ eine Zahl in einem solchen algebraischen Zahlkörper $K$ (vom Grade $n \geqq 3$ ) ist, der die folgende Eigenschaft hat:

Man kennt eine primitive ganze Zahl $\varrho$ aus $K$ und ganze rationale teilerfremde Zahlen $p, q(q>0)$ derart, dass die Bedingung

$$
|\varrho-p| q \mid<1 / q^{*}, \quad *>\varkappa_{0}, \quad q>q_{0}
$$

erfüllt ist, wo $\varkappa_{0}=\varkappa_{0}(\lambda)$ und $q_{0}=q_{0}(\varrho, \varkappa, \lambda)$ gewisse (in [7] bzw. [8] explizit angegebene) Schranken sind.

In [7], wo eine Beweismethode ähnlich wie bei THus [14] angewendet ist, ist $x_{0}=n \lambda /(2 \lambda-2)$. In [8] haben wir durch Anwendung der Ideen von z.B. Gelfond [6], Dyson [5] und Schneider [12] für $\varkappa_{0}$ einen etwas besseren Wert $2 n / \lambda$ erreicht. Eine der Schwächen der genannten Ergebnisse liegt darin, dass $\varkappa$ nahe $n$ sein muss, wenn $\lambda$ nahe 2 ist. Das Hauptziel der vorliegenden Arbeit ist, uns von dieser Schwäche zu befreien, indem wir 
neben den Ideen von [7], [8] Näherungspolynome mehrerer Veränderlichen zu Hilfe nehmen, wie z.B. bei SchneIder [11], Roth und Davenport [10], [4]. In dieser Arbeit können $\varkappa$ und $\lambda$ gleichzeitig nahe an der kritischen Schranke 2 liegen. Dabei muss man jedoch mehrere Approximationen der Form (2) kennen, während es in [7] und [8] reicht, wenn nur eine Approximation (2) bekannt ist. Die vorliegenden Ergebnisse decken also nicht unsere früheren Resultate [7], [8], sondern beleuchten die Frage unter einem anderen Gesichtswinkel.

Eines der Ziele dieser Arbeit (sowie unserer Arbeiten [7], [8]) ist auch, die Aufmerksamkeit darauf zu lenken, dass es eine enge Abhängigkeit zwischen den Approximationen verschiedener Zahlen eines festen algebraischen Zahlkörpers gibt.

2. Formulierung des Hauptergebnisses. Bezeichnungen. Zuerst setzen wir die

Definition. Es sei $K$ ein algebraischer Zahlkörper vom Grade $n \geqq 3$ und $\lambda$ eine reelle Zahl mit $2<\lambda \leqq n$. Wir sagen, dass $K$ die Eigenschaft

$$
E\left(\lambda, m, \varkappa(1), \ldots, \varkappa(m), \vartheta_{1}, \ldots, \vartheta_{m}, q_{1}, \ldots, q_{m}\right)
$$

hat, wenn man folgende Grössen kennt:

- eine natürliche Zahl $m$;

- reelle Zahlen $x(1), \ldots, \varkappa(m)$ mit $2<x(i) \leqq n \quad(i=1, \ldots, m)$;

- ganze irrationale Zahlen $\vartheta_{\mathbf{1}}, \ldots, \vartheta_{m}$ aus $K$, wobei $\vartheta_{1}$ primitiv ist;

- ganze rationale Zahlen $p_{i}, q_{i}$ mit $\left(p_{i}, q_{i}\right)=1, q_{i}>0(i=1, \ldots, m)$ derart, dass die folgenden Bedingungen erfüllt sind:

$$
\begin{aligned}
& 1 / \varkappa(1)+\ldots+1 / \varkappa(m)<(m+1) / 2-((m+1) \log n)^{1 / 2}-1 / \lambda, \\
& \left|\vartheta_{i}-p_{i}\right| q_{i} \mid<1 / q_{i}^{(i)} \\
& (i=1, \ldots, m) \text {, } \\
& q_{1}>g, \quad q_{i+1} \geqq q_{i}^{\beta} \\
& (i=1, \ldots, m-1) \text {, }
\end{aligned}
$$

wobei die Funktionen

$$
\begin{gathered}
\beta=\beta(\lambda, m, \varkappa(1), \ldots, \varkappa(m), n), \\
g=g\left(\lambda, m, \varkappa(1), \ldots, \varkappa(m), \vartheta_{1}, \ldots, \vartheta_{m}\right)
\end{gathered}
$$

wie in (6) und (7) (s. unten) definiert sind.

Unser Hauptresultat ist der

Satz. Es sei $K$ ein algebraischer Zahlkörper vom Grade $n \geqq 3$. Es seien $C, \lambda$ reelle Zahlen mit $C>0,2<\lambda \leqq n$. Der Körper $K$ habe die Eigenschaft 


$$
E\left(\lambda, m, \varkappa(1), \ldots, \varkappa(m), \vartheta_{1}, \ldots, \vartheta_{m}, q_{1}, \ldots, q_{m}\right) .
$$

Dann kann man für jede irrationale Zahl $\vartheta$ des Körpers $K$ eine solche Schranke

$$
G=G\left(\lambda, m, \varkappa(1), \ldots, \varkappa(m), \vartheta_{1}, \ldots, \vartheta_{m}, q_{1}, \ldots, q_{m}, \vartheta, C\right)
$$

explizit (z.B. durch (8), s. unten) angeben, dass die Ungleichung (1) keine Lösungen $u, v$ mit $(u, v)=1$ und $v \geqq G$ hat.

Nun führen wir einige Bezeichnungen ein. Wegen (3) können wir positive Hilfsgrössen $\varepsilon, \zeta, \xi$ wählen derart, dass die Zahl

$$
\Delta=M / 2-\delta-\zeta-(1+\xi)(1 / \varkappa(1)+\ldots+1 / \varkappa(m))-1 / \lambda
$$

mit $M=m+1$ und $\delta=(M \log n)^{1 / 2}+\varepsilon$ positiv ist und zugleich die Ungleichung

$$
\delta \leqq(M \log (2 n))^{1 / 2}
$$

besteht. Weiter setzen wir

$$
\gamma=\zeta \varkappa(1) / 6(1+\xi)^{2} \max (\varkappa(1), \ldots, \varkappa(m)),
$$$$
\log \mu=2^{m-1}(\log \gamma-M \log 2),
$$

$$
\begin{aligned}
& \beta_{1}=3(1+\xi)^{3} \varkappa(2)^{-1} \max (\varkappa(1), \ldots, \varkappa(m)) / 2 \mu, \\
& \beta_{2}=(1+\xi) \max (\varkappa(2) / \varkappa(3), \varkappa(3) / \varkappa(4), \ldots, \varkappa(m-1) / \varkappa(m)) / \mu,
\end{aligned}
$$

$$
\beta=\max \left(\beta_{1}, \beta_{2}, 1+1 / \xi\right) \text {. }
$$

Es sei

$$
f(x)=x^{n}+a_{1} x^{n-1}+\ldots+a_{n}
$$

das kanonische Polynom für $\vartheta_{1}\left(\vartheta_{1}\right.$ ist ja primitiv, also vom Grade $\left.n\right)$, wobei

$$
a=\max \left(\left|a_{1}\right|, \ldots,\left|a_{n}\right|\right)
$$

die Höhe von $\vartheta_{1}$ (in Bezeichnung $H\left(\vartheta_{1}\right)$ ) ist. Es sei

$$
\begin{aligned}
\eta & =n /\left(\exp \left(\delta^{2} / M\right)-n\right), \\
\alpha & =\lambda(1+\xi) / \varkappa(1), \\
\tau & =2 \lambda \mu / 3 \varkappa(1)(1+\xi)(1+\xi-\mu)
\end{aligned}
$$

gesetzt und ferner 
A. I. 394

$$
\begin{aligned}
& C_{1}=n !^{2}(a+1)^{n(n-1)}, \\
& C_{2}=C_{1}^{n+1 / 2} \\
& C_{3}=(2 a+2)^{\alpha}\left(2 C_{2}(2 a+2)^{n-1} \max \left(H\left(\vartheta_{2}\right), \ldots, H\left(\vartheta_{m}\right)\right)\right)^{\tau}, \\
& C_{4}=8^{\alpha} 4^{\tau} C_{3}^{2 \eta}, \\
& C_{5}=\left(4\left|\vartheta_{1}\right|+4\right)^{\alpha}\left(4 C_{1}^{n+1} \max \left(H\left(\vartheta_{2}\right), \ldots, H\left(\vartheta_{m}\right)\right)\right)^{\tau} C_{3}^{\eta}, \\
& C_{6}=C_{3}^{\eta} \\
& C_{7}=C_{4}^{m \varkappa(1) / 2 \hat{\lambda} \mu}, \\
& C_{8}=C_{5}^{1 / \lambda \Delta}, \\
& C_{9}=2^{m(m-1)(2 m+1) / \mu},
\end{aligned}
$$

$$
g=\max \left(C_{1}, C_{6}, C_{7}, C_{8}, C_{9}\right)
$$

Wir setzen $\vartheta=\vartheta_{M}, \quad \lambda=\varkappa(M), u=p_{M}, v=q_{M}$. Für $i=1, \ldots, M$ sei $T_{i}$ die kleinste natürliche Zahl derart, dass die Koeffizienten $d_{i 1}, \ldots, d_{i n}$ in den Ausdrücken

$$
T_{i} \vartheta_{i}=g_{i}\left(\vartheta_{1}\right), \quad g_{i}(x)=d_{i 1} x^{n-1}+\ldots+d_{i n} \quad(i=1, \ldots, M)
$$

ganze rationale Zahlen sind. Dabei seien die Bezeichnungen

$$
T_{i} \vartheta_{i}=\Theta_{i} \quad(i=1, \ldots, M)
$$

eingeführt. (Es ist $T_{1}=1, \Theta_{1}=\vartheta_{1}$.) Weiter setzen wir

$$
\begin{aligned}
& C_{10}=2^{M+n} n !(a+1)^{n(n-1) / 2}\left(H\left(\Theta_{M}\right)+1\right), \\
& C_{11}=C_{1} C_{10}^{\eta} H(\vartheta), \\
& C_{12}=C_{10}^{\eta m \varkappa(1) / / \mu}, \\
& C_{13}=2^{M+1} C_{10}^{\eta}\left(j \Theta_{M} !+1\right) \max \left(1, C C_{1} H(\vartheta)\right), \\
& C_{14}=C_{13}^{1 / \lambda .4}, \\
& C_{15}=\log C_{11} / \log \left(q_{1} / C_{6}\right), \\
& C_{16}=\log C_{12} / \log \left(q_{1} / C_{7}\right), \\
& C_{17}=\log C_{14} / \log \left(q_{1} / \mathrm{C}_{8}\right), \\
& C_{18}=\varkappa(m) \log q_{m} / \lambda \min (\xi, 2 \gamma) \log q_{1},
\end{aligned}
$$

$$
\log G=\max \left(C_{15}, C_{16}, C_{17}, C_{18}\right) \log q_{1} .
$$


3. Beweis des Satzes. Anfangs schreiben wir zwei bekannte Hilfssätze. Unser tiefstes Hilfsmittel ist der folgende, zum wesentlichsten Teil auf den Beweis von Rotr [10] begründete Hilfssatz 1, der Polynome von der Form

$$
A\left(x_{1}, \ldots, x_{m}\right)=\sum_{i(1)=0}^{s(1)} \ldots \sum_{i(m)=0}^{s(m)} a_{i(1) \ldots i(m)} x_{1}^{i(1)} \ldots x_{m}^{i(m)}
$$

behandelt. Dabei setzen wir

$$
\begin{gathered}
A_{j(1) \ldots j(m)}\left(x_{1}, \ldots, x_{m}\right) \\
=\partial^{j(1)+\ldots+j(m)} A\left(x_{1}, \ldots, x_{m}\right) / j(1) ! \ldots j(m) ! \partial x_{1}^{j(1)} \ldots \partial x_{m}^{j(m)}
\end{gathered}
$$

für ganze rationale, nicht negative $j(1), \ldots, j(m)$.

Hilfssatz 1. Es sei $m$ eine natürliche Zahl $\geqq 2$. Es seien $\mu, S$ reelle Zahlen, $0<\mu \leqq 1, \quad S \geqq 1$, und $s(1), \ldots, s(m)$ natürliche Zahlen mit

$$
s(i+1) \leqq s(i) \mu \quad(i=1, \ldots, m-1) .
$$

Für $i=1, \ldots, m$ seien $u_{i}, v_{i}$ ganze rationale Zahlen mit $\left(u_{i}, v_{i}\right)=1$, $v_{i}>0$ und

$$
\begin{array}{cl}
s(i) \log v_{i} \geqq s(1) \log v_{1} & (i=2, \ldots, m), \\
v_{1} \geqq 2^{m(m-1)(2 m+1) / \mu}, & \\
v_{1}^{s(1) u / m} \geqq S . &
\end{array}
$$

Die Koeffizienten $a_{i(1) \ldots i(m)}$ des Polynoms (9) seien ganze rationale, nicht sämtlich verschwindende Zahlen, die absolut genommen höchstens gleich $S$ sind. Dann gibt es ganze rationale, nicht negative Zahlen $j(1), \ldots, j(m)$ derart, dass die Ungleichungen

und

$$
A_{j(1) \ldots j(m)}\left(u_{1} / v_{1}, \ldots, u_{m} / v_{m}\right) \neq 0
$$

$$
j(1) / s(1)+\ldots+j(m) / s(m) \leqq \gamma
$$

mit $\log \gamma=(m+1) \log 2+2^{1-m} \log \mu$ bestehen.

Beweis. Der Hilfssatz folgt leicht aus den Betrachtungen von MaHLer. Vgl. [9], S. 97, Th. 1 und den Beweis.

Die wesentliche Idee des folgenden Hilfssatzes rührt von ScHNEIDER [11] her.

Hilfssatz 2. Es sei $M$ eine natürliche und $\delta$ eine positive Zahl. Es seien $r(1), \ldots, r(M)$ natürliche Zahlen. Dann ist die Anzahl der Gitterpunkte $(j(1), \ldots, j(M))$ mit den Eigenschaften 


$$
0 \leqq j(i) \leqq r(i)
$$

$(i=1, \ldots, M)$

und

$$
j(1) / r(1)+\ldots+j(M) / r(M)<M / 2-\delta
$$

höchstens gleich

$$
(r(1)+1) \ldots(r(M)+1) \exp \left(-\delta^{2} / M\right) .
$$

Beweis. Vgl. Mahler [9], S. 166, Th. 1.

Von nun an werden die Bezeichnungen von Nr. 2 angewendet. Dazu führen wir noch einige Bezeichnungen ein. Gegen den Hauptsatz sei angenommen, dass die Ungleichung (1) eine Lösung $u, v$ mit $(u, v)=1$ und $v \geqq G$ besitzt.

Wir setzen

$$
T_{i} p_{i} / q_{i}=u_{i} / v_{i} \quad(i=1, \ldots, M),
$$

wobei $u_{i}, v_{i}$ ganze rationale Zahlen mit $\left(u_{i}, v_{i}\right)=1$ und $v_{i}>0$ sind. Wie in [7] (vgl. [7], Hilfssatz 1, oder [8], Hilfssatz 2) sehen wir, dass die Zahlen $T_{2}, \ldots, T_{m}$ kleiner als $C_{1}$ sind. Bezeichnet $T$ die kleinste natürliche Zahl derart, dass die Zahl $T \vartheta$ ganz ist, so können wir eine natürliche Zahl $T^{\prime}$ mit $T^{\prime}<C_{1}$ wählen, so dass in der kanonischen Darstellung von $T^{\prime} T \vartheta$ als Polynom von $\vartheta_{1}$ die Koeffizienten ganz rational sind. Da also $T_{M} \leqq T^{\prime} T$ und ferner $T \leqq H(\vartheta)$ ist, so gilt die Abschätzung $T_{M}<C_{1} H(\vartheta)$. Aus den Ungleichungen (1) und (4) folgen nun die Ungleichungen

$$
\left|\Theta_{i}-u_{i}\right| v_{i}\left|<C^{(i)}\right| q_{i}^{\varkappa(i)} \quad(i=1, \ldots, M)
$$

wobei

$$
C^{(1)}=1, \quad C^{(1 I)}=C C_{1} H(\vartheta), \quad C^{(i)}=C_{1} \quad(i=2, \ldots, m)
$$

gesetzt ist. Weiter gilt wegen $\left(p_{i}, q_{i}\right)=1$ die Abschätzung

$$
v_{M}>q_{M} / C_{1} H(\vartheta), \quad v_{i}>q_{i} / C^{(i)} \quad(i=2, \ldots, m) .
$$

Ferner setzen wir

$$
\sigma(i)=\log q_{i} / \log q_{1} \quad(i=1, \ldots, M),
$$

also $\sigma(1)=1$. Ausserdem wird auch kurz $\sigma(M)=\sigma$ geschrieben. Wir wählen ganze rationale Zahlen $r(1), \ldots, r(M)$ unter den Bedingungen

$$
\sigma \lambda / \sigma(i) \varkappa(i) \leqq r(i)<\sigma \lambda / \sigma(i) \varkappa(i)+1 \quad(i=1, \ldots, M) .
$$

Also ist $r(M)=1$. Nach (5) ist 


$$
\sigma(i+1) \geqq \beta \sigma(i) \quad(i=1, \ldots, m-1) .
$$

Daher gilt für $i=2, \ldots, m-1$

$$
\sigma(i+1) \varkappa(i+1) \geqq \beta_{2} \sigma(i) \varkappa(i+1) \geqq(1+\xi) \sigma(i) \varkappa(i) / \mu,
$$

also, wegen $\mu<1, \sigma(i+1) \varkappa(i+1)>\sigma(i) \varkappa(i)$. Es gilt auch

$$
\sigma(2) \varkappa(2) \geqq \beta_{1} \sigma(1) \varkappa(2) \geqq 3(1+\xi)^{3} \sigma(1) \varkappa(1) / 2 \mu,
$$

also $\sigma(2) \varkappa(2)>\sigma(1) \varkappa(1)$. Da weiter nach $v \geqq G$ die Ungleichung $\sigma \geqq C_{18}$, folglich die Ungleichung $\sigma \geqq \sigma(m) \varkappa(m) / \lambda \xi$ besteht, so ergibt sich die Abschätzung

$$
\sigma \lambda \leqq r(i) \sigma(i) \varkappa(i)<\sigma \lambda(1+\xi) \quad(i=1, \ldots, M) .
$$

Hierbei sei auch bemerkt, dass die Zahlenfolge $r(1), \ldots, r(M)$ monoton fallend ist.

Wir setzen noch

$$
\alpha(i)=\lambda(1+\xi) / \sigma(i) \varkappa(i) \quad(i=1, \ldots, m) .
$$

Also ist $\alpha(1)=\alpha$ und nach (17)

$$
r(i)<\sigma \alpha(i) \quad(i=1, \ldots, m) .
$$

Nach den obigen Folgerungen von (16) gilt

$$
\begin{aligned}
& \alpha(i+1) \leqq \alpha(i) \mu /(1+\xi) \quad(i=2, \ldots, m-1), \\
& \alpha(2) \leqq 2 \alpha(1) \mu / 3(1+\xi)^{3},
\end{aligned}
$$

folglich

$$
\begin{gathered}
\alpha(2)+\ldots+\alpha(m)<\alpha(2)\left(1+\mu /(1+\xi)+(\mu /(1+\xi))^{2}+\ldots\right) \\
\leqq 2 \alpha(1) \mu / 3(1+\xi)^{2}(1+\xi-\mu) .
\end{gathered}
$$

Daraus ergibt sich die Abschätzung

$$
r(1)<\sigma \alpha, \quad r(2)+\ldots+r(m)<\sigma \tau .
$$

In unserem Beweis spielt eine zentrale Rolle ein Polynom von der Form

$$
P\left(x_{1}, \ldots, x_{M}\right)=\sum_{i(1)=0}^{r(1)} \ldots \sum_{i(M)=0}^{r(M)} c_{i(1)} \ldots i(M) x_{1}^{i(1)} \ldots x_{M}^{i(M)} .
$$

Die Koeffizienten $c_{i(1)} \ldots i(M)$ werden später wie in Hilfssatz 4 gewählt. Für ganze rationale, nicht negative $j(1), \ldots, j(M)$ schreiben wir

$$
\begin{gathered}
P_{j(1) \ldots j(M)}\left(x_{1}, \ldots, x_{M}\right) \\
=\partial^{j(1)+\ldots+j(M)} P\left(x_{1}, \ldots, x_{M}\right) / j(1) ! \ldots j(M) ! \partial x_{1}^{j(1)} \ldots \partial x_{M}^{j(M)} .
\end{gathered}
$$


Hilfssatz 3. Es seien $j(1), \ldots, j(M)$ ganze rationale Zahlen $\geqq 0$. Dann besteht die Gleichung

$$
\begin{gathered}
P_{j(1) \ldots j(M)}\left(\Theta_{1}, \ldots, \Theta_{M}\right) \\
=\sum_{i=0}^{n-1} \sum_{i(1)=0}^{r(1)} \ldots \sum_{i(M)=0}^{r(M)} B(j(1), \ldots, j(M), i, i(1), \ldots, i(M)) c_{i(1) \ldots i(M)} \vartheta_{1}^{i},
\end{gathered}
$$

wobei die Koeffizienten $B(j(1), \ldots, j(M), i, i(1), \ldots, i(M))$ ganze rationale Zahlen sind und für alle $j(1), \ldots, j(M), i$ der Bedingung

$$
\sum_{i(1)=0}^{r(1)} \ldots \sum_{i(M)=0}^{r(M)}|B(j(1), \ldots, j(M), i, i(1), \ldots, i(M))|<C_{3}^{\sigma} C_{10}-1
$$

genügen.

Beweis. (Vgl. [8], Hilfssatz 4.) Für $j=2, \ldots, M$ gilt

$$
g_{j}(x) \ll d_{j}(x+1)^{n-1}
$$

mit $d_{j}=\max \left(\left|d_{j 1}\right|, \ldots,\left|d_{j n}\right|\right)$, wobei die Bezeichnung $\ll$ bedeutet, dass jeder Koeffizient des auf der linken Seite stehenden Polynoms absolut genommen höchstens gleich dem entsprechenden Koeffizienten auf der rechten Seite ist und kein Koeffizient auf der rechten Seite negativ ist. Also ist

$$
\begin{gathered}
g_{2}(x)^{i(2)} \ldots g_{M}(x)^{i(M)} \\
\ll d_{2}^{i(2)} \ldots d_{M}^{i(M)} 2^{(n-1)(i(2)+\ldots+i((M))}\left(x^{(n-1)(i(2)+\cdots+i(M))}+\ldots+x+1\right) .
\end{gathered}
$$

Im Moment sei $t=i(1)+(n-1)(i(2)+\ldots+i(M))$. Nun können wir

$$
\Theta_{1}^{i(1)} \ldots \Theta_{M}^{i(M)}=g_{1}\left(\vartheta_{1}\right)^{i(1)} \ldots g_{M}\left(\vartheta_{1}\right)^{i(M)}=\sum_{i=0}^{t} c_{i} \vartheta_{1}^{i}
$$

schreiben, wobei die ganzen rationalen Koeffizienten $c_{i}$ absolut genommen höchstens gleich

$$
d_{2}^{i(2)} \ldots d_{M}^{i(M)} 2^{(n-1)(i(2)+\cdots+i(M))}
$$

sind. Durch wiederholte Anwendung der Beziehung $f\left(\vartheta_{1}\right)=0$ sehen wir jetzt, dass in dem Ausdruck

$$
\Theta_{1}^{i(1)} \ldots \Theta_{M}^{i(M)}==\sum_{i=0}^{n-1} b(i(1), \ldots, i(M), i) \vartheta_{1}^{i}
$$

die Koeffizienten $b(i(1), \ldots, i(M), i)$ ganze rationale Zahlen sind, die für $i(1) \geqq n$ und für $i(2)+\ldots+i(M) \geqq 1$ absolut genommen nicht grösser als

$$
d_{2}^{i(2)} \ldots d_{M}^{i(M)} 2^{(n-1)(i(2)+\ldots+i(M))}(a+1)^{t-(n-1}
$$


sind. Ist $i(1)<n$, so ist $b(i(1), 0, \ldots, 0, i)$ für $i=i(1)$ gleich 1 und sonst gleich Null. Weiter gilt

$$
\begin{gathered}
P_{j(1) \ldots j(M)}\left(\Theta_{1}, \ldots, \Theta_{M}\right) \\
=\sum^{*}\left(\begin{array}{l}
i(1) \\
j(1)
\end{array}\right) \ldots\left(\begin{array}{l}
i(M) \\
j(M)
\end{array}\right) c_{i(1) \ldots i(M)} \Theta_{1}^{i(1)-j(1)} \ldots \Theta_{M}^{i(M)-j(M)} \\
=\sum_{i=0}^{n-1} \sum^{*}\left(\begin{array}{l}
i(1) \\
j(1)
\end{array}\right) \ldots\left(\begin{array}{l}
i(M) \\
j(M)
\end{array}\right) b(i(1)-j(1), \ldots, i(M)-j(M), i) c_{i(1) \ldots i(M)} \vartheta_{1}^{i}
\end{gathered}
$$

mit der Bezeichnung

$$
\Sigma^{*}=\sum_{i(1)=j(1)}^{r(1)} \cdots \sum_{i(M)=j(M)}^{r(M)} .
$$

Hierbei besteht nach dem oben Bewiesenen die Abschätzung

$$
\sum^{*}\left(\begin{array}{l}
i(1) \\
j(1)
\end{array}\right) \ldots\left(\begin{array}{l}
i(M) \\
j(M)
\end{array}\right)|b(i(1)-j(1), \ldots, i(M)-j(M), i)|
$$

$<2^{r(1)}+(a+1)^{1-n} \sum *(2 a+2)^{i(1)}\left(2 d_{2}(2 a+2)^{n-1}\right)^{i(2)} \ldots\left(2 d_{M}(2 a+2)^{n-1}\right)^{i(M)}$ $<2^{M}(a+1)^{1-n}(2 a+2)^{r(1)}\left(2 d_{2}(2 a+2)^{n-1}\right)^{r(2)} \ldots\left(2 d_{M}(2 a+2)^{n-1}\right)^{r(M)}$.

Wie in [7] (vgl. [7], Hilfssatz 1, oder [8], Hilfssatz 2) bekommen wir die Abschätzungen

$$
d_{j}<n !(a+1)^{n(n-1) / 2}\left(H\left(\Theta_{j}\right)+1\right) \quad(j=2, \ldots, M) .
$$

Für $j=M$ wenden wir diese Abschätzung an. Für $j=2, \ldots, m$ gilt, wie schon erwähnt, $T_{j}<C_{1}$, also weiter

$$
H\left(\Theta_{j}\right)+1 \leqq T_{j}^{n} H\left(\vartheta_{j}\right)+1<C_{1}^{n} H\left(\vartheta_{j}\right)
$$

Daraus ergibt sich

$$
d_{j}<C_{2} H\left(\vartheta_{j}\right) \quad(j=2, \ldots, m) .
$$

Folglich ist die Summe (22) wegen $r(M)=1$ kleiner als

$$
C_{10}(2 a+2)^{r(1)}\left(2 C_{2} H\left(\vartheta_{2}\right)(2 a+2)^{n-1}\right)^{r(2)} \ldots\left(2 C_{2} H\left(\vartheta_{m}\right)(2 a+2)^{n-1}\right)^{r(m)} .
$$

Hieraus folgt nach den Ungleichungen (18) eine Abschätzung von der Form (20). Damit ist Hilfssatz 3 bewiesen.

Hilfssatz 4. Es gibt ein Polynom der Form (19), das die folgenden Eigenschaften besitzt:

Die Koeffizienten $c_{i(1) \ldots i(M)}$ sind ganze rationale, nicht sämtlich verschwindende Zahlen, die absolut genommen kleiner als $C_{6}^{\sigma} C_{10}^{n}$ sind. 
Die Gleichung

$$
P_{j(1) \ldots j(M)}\left(\Theta_{1}, \ldots, \Theta_{M}\right)=0
$$

besteht für alle ganzen rationalen $j(1), \ldots, j(M)$ mit

(23) $j(1) / r(1)+\ldots+j(M) / r(M)<M / 2-\delta, 0 \leqq j(i) \leqq r(i) \quad(i=1, \ldots, M)$.

Beweis. (Vgl. [8], Hilfssatz 5.) Nach Hilfssatz 3 ist die letztere Eigenschaft äquivalent mit dem Gleichungssystem

$$
\sum_{i(1)=0}^{r(1)} \ldots \sum_{i(M)=0}^{r(M)} B(j(1), \ldots, j(M), i, i(1), \ldots, i(M)) c_{i(1) \ldots i(M)}=0,
$$

wobei $j(1), \ldots, j(M), i$ alle ganzen rationalen Werte mit (23) und $0 \leqq i<n$ annehmen. Hierin ist die Anzahl der Gleichungen nach Hilfssatz 2 höchstens gleich

$$
(r(1)+1) \ldots(r(M)+1) \eta /(1+\eta) .
$$

Mittels des Schubfachschlusses (s. [8], Hilfssatz 1, oder [13], S. 8) wegen (20) sehen wir jetzt, dass man solche ganze rationale, nicht sämtlich verschwindende Koeffizienten $c_{i(1) \ldots i(M)}$ wählen kann, die absolut genommen kleiner als

$$
\left(C_{3}^{\sigma} C_{10}-1\right)^{n}+1
$$

also wegen $\eta \geqq 1$ kleiner als $C_{3}^{\eta \sigma} C_{10}^{\eta}$ sind. Daraus folgt Hilfssatz 4 .

Von jetzt an ist vorausgesetzt, dass das Polynom (19) die Eigenschaften des Hilfssatzes 4 hat.

Hilfssatz 5. Es gibt ganze rationale, nicht negative Zahlen $j(1), \ldots, j(m)$ mit

und

$$
P_{j(1) \ldots j(m) 0}\left(u_{1} / v_{1}, \ldots, u_{M} / v_{M}\right) \neq 0
$$

$$
j(1) / r(1)+\ldots+j(m) / r(m)<\zeta .
$$

Beweis. Das Polynom (19) lässt sich in der Form

$$
P\left(x_{1}, \ldots, x_{M}\right)=x_{M} Q\left(x_{1}, \ldots, x_{m}\right)+R\left(x_{1}, \ldots, x_{m}\right)
$$

darstellen. Für $k=1, \ldots, m$ setzen wir

$$
\begin{gathered}
W_{k}\left(x_{1}, \ldots, x_{m}\right) \\
=Q\left(x_{1}, \ldots, x_{m}\right) \partial R\left(x_{1}, \ldots, x_{m}\right) / \partial x_{k}-R\left(x_{1}, \ldots, x_{m}\right) \partial Q\left(x_{1}, \ldots, x_{m}\right) / \partial x_{k} .
\end{gathered}
$$


Erstens sei angenommen, dass es einen Wert von $k$ gibt, für den das Polynom $W_{k}\left(x_{1}, \ldots, x_{m}\right)$ nicht identisch verschwindet. Für ein solches $k$ schreiben wir

$$
A\left(x_{1}, \ldots, x_{m}\right)=W_{k}\left(x_{1}, \ldots, x_{m}\right)
$$

und wenden Hilfssatz 1 an. Da nun der Grad des Polynoms $A$ in bezug auf $x_{i} \quad(i=1, \ldots, m)$ höchstens gleich $2 r(i)$ ist, können wir in (9) $s(1)=2 r(1)$ und

$$
s(i)=\left[3(1+\xi)^{2} x(1)^{-1} \max (\varkappa(1), \ldots, x(m))\right] r(i) \quad(i=2, \ldots, m)
$$

setzen. Die Eigenschaft (10) folgt dann aus den nach (17), (16) und (6) geltenden Abschätzungen

$$
\begin{gathered}
s(2) / s(1)<3(1+\xi)^{2} \varkappa(1)^{-1} \max (x(1), \ldots, x(m))(1+\xi) \varkappa(1) / 2 \beta x(2) \\
=\mu \beta_{1} / \beta \leqq \mu
\end{gathered}
$$

und

$s(i+1) / s(i)<(1+\xi) \varkappa(i) / \beta \varkappa(i+1) \leqq \mu \beta_{2} / \beta \leqq \mu \quad(i=2, \ldots, m-1)$.

Nach (17) besteht die Ungleichung

$$
s(1) \log v_{1}=2 r(1) \log q_{1}<2(1+\xi) \varkappa(1)^{-1} \varkappa(i) r(i) \sigma(i) \log q_{1} .
$$

Da nach (5), (7) $q_{1}>C_{1}$ ist, so gilt wegen (15) die Abschätzung

$$
(\sigma(i)-1) \log q_{1}<\log v_{i} \quad(i=2, \ldots, m) .
$$

Nach (16) und (6) gilt weiter

$$
1-1 / \sigma(i) \geqq 1-1 / \beta \geqq 1 /(1+\xi) \quad(i=2, \ldots, m) .
$$

Daher ist $\sigma(i) \log q_{1}<(1+\xi) \log v_{i}$ und also

$$
s(1) \log v_{1}<2(1+\xi)^{2} \varkappa(1)^{-1} \max (\varkappa(1), \ldots, \varkappa(m)) r(i) \log v_{i}<s(i) \log v_{i}
$$

für $i=2, \ldots, m$, womit (11) bewiesen ist. Die Eigenschaft (12) folgt aus $q_{1}>C_{9}$.

Weil die Koeffizienten der Polynome $Q$ und $R$ auch Koeffizienten des Polynoms $P$ sind, gilt nach der Wahl von $P$

$$
Q\left(x_{1}, \ldots, x_{m}\right), R\left(x_{1}, \ldots, x_{m}\right) \ll C_{6}^{\sigma} C_{10}^{\prime}\left(x_{1}+1\right)^{r(1)} \ldots\left(x_{m}+1\right)^{r(m)}
$$

und daher

$$
A\left(x_{1}, \ldots, x_{m}\right) \ll 2 r(k) C_{6}^{2 \sigma} C_{10}^{2 \eta}\left(x_{1}+1\right)^{2 r(1)} \ldots\left(x_{m}+1\right)^{2 r(m)} .
$$

Folglich sind die Koeffizienten des Polynoms $A$ absolut genommen kleiner als

$$
2^{3 r(1)+2(r(2)+\cdots+r(m))} C_{6}^{2 \sigma} C_{10}^{2 \eta} .
$$


Nach (18) können wir also $S=C_{4}^{\sigma} C_{10}^{2 \eta}$ wählen; die Bedingung (13) ist nämlich wegen $q_{1}>C_{7}$ und $\sigma \geqq C_{16}$ erfüllt.

Aus Hilfssatz 1 folgt nun die Existenz solcher ganzen rationalen, nicht negativen Zahlen $k(1), \ldots, k(m)$, dass die Ungleichungen

und

$$
A_{k(1) \ldots k(m)}\left(u_{1} / v_{1}, \ldots, u_{m} / v_{m}\right) \neq 0
$$

$$
k(1) / s(1)+\ldots+k(m) / s(m) \leqq \gamma
$$

bestehen. Also gibt es auch ganze rationale, nicht negative Zahlen $h(i)$, $j(i) \quad(i=1, \ldots, m)$ derart, dass

$$
\begin{aligned}
& Q_{h(1) \ldots h(m)}\left(u_{1} / v_{1}, \ldots, u_{m} / v_{m}\right) R_{j(1) \ldots j(m)}\left(u_{1} / v_{1}, \ldots, u_{m} / v_{m}\right) \\
\neq & R_{h(1) \ldots h(m)}\left(u_{1} / v_{1}, \ldots, u_{m} / v_{m}\right) Q_{j(1) \ldots j(m)}\left(u_{1} / v_{1}, \ldots, u_{m} / v_{m}\right)
\end{aligned}
$$

ist und dabei wegen $s(m) \leqq s(i) \quad(i=1, \ldots, m)$ die Ungleichungen

$$
\begin{aligned}
& h(1) / s(1)+\ldots+h(m) / s(m) \leqq \gamma+1 / s(m), \\
& j(1) / s(1)+\ldots+j(m) / s(m) \leqq \gamma+1 / s(m)
\end{aligned}
$$

bestehen.

Verschwinden jetzt die beiden Polynome $P_{h(1) \ldots h(m) 0}$ und $P_{j(1) \ldots j(m) 0}$ im "Punkt» $x_{1}=u_{1} / v_{1}, \ldots, x_{M}=u_{M} / v_{M}$, so verschwindet auch das Polynom

$$
\begin{aligned}
& Q_{h(1) \ldots h(m)} P_{j(1) \ldots j m) 0}-Q_{j(1) \ldots j(m)} P_{h(1) \ldots h(m) 0} \\
= & Q_{h(1) \ldots h(m)} R_{j(1) \ldots j(m)}-R_{h(1) \ldots h(m)} Q_{j(1) \ldots j(m)}
\end{aligned}
$$

in diesem Punkt gegen (24). Also ist das Polynom $P_{j(1) \ldots j(m) 0}$ im genannten Punkt von Null verschieden, wenn nötigenfalls die Rollen von den $h(i)$ und $j(i)$ getauscht sind. Wegen (17) und $\sigma \geqq C_{18}$ ist

also

$$
\gamma+1 / s(m)<\gamma+1 / 2 r(m) \leqq \gamma+\sigma(m) \varkappa(m) / 2 \sigma \lambda \leqq 2 \gamma,
$$

$j(1) / r(1)+\ldots+j(m) / r(m)<6 \gamma(1+\xi)^{2} \varkappa(1)^{-1} \max (\varkappa(1), \ldots, \varkappa(m))=\zeta$, womit der Hilfssatz im vorliegenden Fall bewiesen ist.

Zweitens sei angenommen, dass das Polynom $W_{k}^{*}\left(x_{1}, \ldots, x_{m}\right)$ für jedes $k=1, \ldots, m$ identisch verschwindet. Dann gilt identisch

a) $Q\left(x_{1}, \ldots, x_{m}\right)=0$ oder

b) $R\left(x_{1}, \ldots, x_{m}\right)=C^{*} Q\left(x_{1}, \ldots, x_{m}\right)$,

wobei $C^{*}$ eine rationale Konstante bedeutet, deren Nenner in gekürzter Form nach den Eigenschaften des Polynoms $P$ kleiner als $C_{6}^{\sigma} C_{10}^{\prime \prime}$ sein muss. Also besteht identisch entweder

a) die Gleichung $P=R$ oder

b) die Gleichung $P=\left(x_{M}+C^{*}\right) Q$. 
Nun führt Hilfssatz 1 mit $S=C_{6}^{\sigma} C_{10}^{n}$ und a) $A=R$ oder b) $A=Q$ leicht zum Ziel (vgl. den ersteren Teil des Beweises). Ausserdem ist festzustellen, dass der Faktor $x_{M}+C^{*}$ für $x_{M}=u_{M} / v_{M}$ nicht verschwindet. Dies folgt (auch für $C^{*}=0=0 / 1$ ) wegen $\left(u_{M}, v_{M}\right)=1$ aus der nach (15), $q_{1}>C_{6}$ und $\sigma \geqq C_{15}$ geltenden Abschätzung

$$
v_{M}>q_{1}^{\sigma} / C_{1} H(\vartheta) \geqq C_{6}^{\sigma} C_{10}^{\eta} .
$$

Damit ist die Richtigkeit des Hilfssatzes 5 festgestellt.

Hilfssatz 6. Es seien $j(1), \ldots, j(m)$ ganze rationale Zahlen $\geqq 0$. Dann gilt die Abschätzung

$$
\begin{gathered}
\left|P_{j(1) \ldots j(m) 0}\left(u_{1} / v_{1}, \ldots, u_{M} / v_{M}\right)\right| \\
<C_{5}^{\sigma} C_{13} q_{1}^{-\sigma \lambda(M / 2-\delta-(j(1)) r(1)+\ldots+j(m) / r(m)))} .
\end{gathered}
$$

Beweis. Der Beweis stützt sich mit $j(M)=0$ auf die Identität

$$
\begin{gathered}
P_{j(1) \ldots j(M)}\left(x_{1}, \ldots, x_{M}\right) \\
=\sum * P_{i(1) \ldots i(M)}\left(\Theta_{1}, \ldots, \Theta_{M}\right)\left(\begin{array}{c}
i(1) \\
j(1)
\end{array}\right) \ldots\left(\begin{array}{c}
i(M) \\
j(M)
\end{array}\right) \times \\
\times\left(x_{1}-\Theta_{1}\right)^{i(1)-j(1)} \ldots\left(x_{M}-\Theta_{M}\right)^{i(M)-j(M)},
\end{gathered}
$$

wobei die Bezeichnung (21) angewendet ist.

Da

$$
P\left(x_{1}, \ldots, x_{M}\right) \ll C_{6}^{\sigma} C_{10}^{n}\left(x_{1}+1\right)^{r_{1}(1)} \ldots\left(x_{M}+1\right)^{r(M)}
$$

ist, so ist

$$
\begin{gathered}
P_{i(1) \ldots i(M)}\left(x_{1}, \ldots, x_{M}\right) \\
\ll C_{6}^{\sigma} C_{10}^{n} 2^{r(1)+\ldots+r(m)}\left(x_{1}+1\right)^{r(1)-i(1)} \ldots\left(x_{M}+1\right)^{r(M)-i(M)} .
\end{gathered}
$$

Daraus ergibt sich die Abschätzung

$$
\left|P_{i(1) \ldots i(M)}\left(\Theta_{1}, \ldots, \Theta_{M}\right)\right| \leqq Z_{1}
$$

mit der Bezeichnung

$$
Z_{1}=C_{6}^{\sigma} C_{10}^{\eta} 2^{r(1)+\cdots+r(m)}\left(\left|\Theta_{1}\right|+1\right)^{r(1)} \ldots\left(\left|\Theta_{m}\right|+1\right)^{r(m)}\left(\left|\Theta_{M}\right|+1\right) .
$$

Da die Summe der obigen Binomialkoeffizientenprodukte kleiner als $2^{M+r(1)+\cdots+r(M)}$ ist, so besteht nach dem letzteren Teil von Hilfssatz 4 die Ungleichung

mit

$$
\left|P_{j(1) \ldots j(M)}\left(u_{1} / v_{1}, \ldots, u_{M} / v_{M}\right)\right|<2^{M+r(1)+\cdots+r(M)} Z_{1} Z_{2}
$$




$$
Z_{2}=\max \left\{\left|u_{1} / v_{1}-\Theta_{1}\right|^{i(1)-j(1)} \cdots\left|u_{M}\right| v_{M}-\left.\Theta_{M}\right|^{i(M)-j(M)}\right\},
$$

wobei $i(1), \ldots, i(M)$ ihre Werte mit der Eigenschaft

$$
i(1) / r(1)+\ldots+i(M) / r(M) \geqq M / 2-\delta
$$

durchlaufen. Im Moment sei $i(1), \ldots, i(M)$ ein solches System, für das das Maximum erreicht ist. Nach (14) gilt dann die Abschätzung

$$
\begin{aligned}
& Z_{2}<\left(C^{(1)} / q_{1}^{\varkappa(1)}\right)^{i(1)-j(1)} \ldots\left(C^{(M)} / q_{M}^{\varkappa(M)}\right)^{i(M)-j(M)}
\end{aligned}
$$

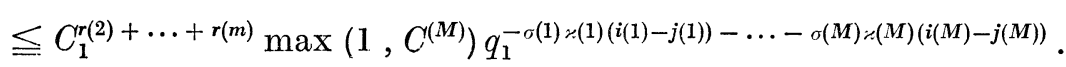

Hierbei ist der Exponent von $q_{1}$ nach (17) höchstens gleich

$$
\begin{aligned}
& -\sigma \lambda((i(1)-j(1)) / r(1)+\ldots+(i(M)-j(M)) / r(M)) \\
& \leqq-\sigma \lambda(M / 2-\delta-(j(1) / r(1)+\ldots+j(m) / r(m))) .
\end{aligned}
$$

Für $i=2, \ldots, m$ gilt weiter wegen $T_{i}<C_{1}$

$$
\left|\Theta_{i}\right|+1<H\left(\Theta_{i}\right)+2 \leqq T_{i}^{n} H\left(\vartheta_{i}\right)+2<C_{1}^{n} H\left(\vartheta_{i}\right) .
$$

Daher ist die Zahl

$$
2^{M+r(1)+\cdots+r(M)} Z_{1} C_{1}^{r(2)+\cdots+r(m)} \max \left(1, C^{(M)}\right)
$$

kleiner als

$$
C_{13} C_{6}^{\tau}\left(4\left|\vartheta_{1}\right|+4\right)^{r(1)}\left(4 C_{1}\right)^{r(2)+\cdots+r(m)}\left(C_{1}^{n} H\left(\vartheta_{2}\right)\right)^{r(2)} \ldots\left(C_{1}^{n} H\left(\vartheta_{m}\right)\right)^{r(m)},
$$

also wegen (18) kleiner als $C_{5}^{\sigma} C_{13}$. Somit ist Hilfssatz 6 bewiesen.

Der Beweis des Satzes wird jetzt mittels der Hilfssätze 5 und 6 beendet. Die Zahlen $j(1), \ldots, j(m)$ seien wie in Hilfssatz 5 gewählt. Dann ist die ganze rationale Zahl

$$
Z_{3}=q_{1}^{r(1)} \ldots q_{M}^{r(M)} P_{j(1) \ldots j(m) 0}\left(u_{1} / v_{1}, \ldots, u_{M} / v_{M}\right)
$$

von Null verschieden, woraus die Abschätzung

$$
\left|Z_{3}\right| \geqq 1
$$

folgt.

Andererseits ergibt sich aus Hilfssatz 6 mit Rücksicht auf die Wahl der Zahlen $j(1), \ldots, j(m)$ die Abschätzung

$$
\left|Z_{3}\right|<C_{5}^{\sigma} C_{13} q_{1}^{\sigma(1) r(1)+\cdots+\sigma(M) r(M)-\sigma \lambda(M / 2-\delta-\zeta)} .
$$

Dabei ist der Exponent von $q_{1}$ nach (17) höchstens gleich 
$-\sigma \lambda(M / 2-\delta-\zeta)+\sigma \lambda(1+\xi)(1 / x(1)+\ldots+1 / \varkappa(m))+\sigma=-\sigma \lambda \Delta$.

Da wegen $q_{1}>C_{8}$ und $\sigma \geqq C_{17}$ die Ungleichung

$$
C_{5}^{\sigma} C_{13} q_{1}^{-\sigma \lambda . \Delta} \leqq 1
$$

besteht, so ist $\left|Z_{3}\right|<1$, was im Widerspruch zu (25) steht.

Der Beweis unseres Hauptsatzes ist damit beendet.

Universität Oulu, Finnland

und

Universität Turku, Finnland 


\section{Literatur}

[1] Baker, A.: Rational approximations to certain algebraic numbers. - Proc. London Math. Soc. (3) 14 (1964), 385-398.

[2] -》- Rational approximations to $\sqrt[3]{\overline{2}}$ and other algebraic numbers. - Quart. J. Math. Oxford (2) 15 (1964), 375-383.

[3] Chowla, S.: The Riemann hypothesis and Hilbert's tenth problem. Blackie, London and Glasgow 1965.

[4] Davenport, H. and Roth, K. F.: Rational approximations to algebraic numbers. - Mathematika 2 (1955), 160-167.

[5] Dyson, F. J.: The approximation to algebraic numbers by rationals. - Acta Math. 79 (1947), 225-240.

[6] Gelfond, A. O.: Transcendental and algebraic numbers. Dover, New York 1960.

[7] Hyxnö, S.: Über Approximation algebraischer Zahlen durch rationale. - Ann. Univ. Turkuensis, Ser. A I, 84 (1965), 1-12.

[8] -»- Über rationale Näherungswerte algebraischer Zahlen. - Ann. Acad. Sci. Fenn., Ser. A I, 376 (1965), 1-15.

[9] Mahler, K.: Lectures on Diophantine approximations. Part 1: g-adic numbers and Roth's theorem. Univ. of Notre Dame 1961.

[10] Rотн, K. F.: Rational approximations to algebraic numbers. - Mathematika 2 (1955), 1-20. Corrigendum. - Mathematika 2 (1955), 168.

[11] SchneIder, Th.: Über die Approximation algebraischer Zahlen. - J. reine angew. Math. 175 (1936), 182-192.

[12] -》- Über eine Dysonsche Verschärfung des Siegel-Thueschen Satzes. - Arch. d. Math. 1 (1948/49), 288-295.

[13] Siegel, C. L.: Über einige Anwendungen diophantischer Approximationen. Abh. Preuss. Akad. Wiss., Phys.-Math. Kl. 1929, Nr. 1, 1-70.

[14] Thus, A.: Über Annäherungswerte algebraischer Zahlen. - J. reine angew. Math. 135 (1909), $284-305$. 